\title{
Measurement of Intraoperative Brain Surface Deformation Under a Craniotomy
}

\author{
Calvin R. Maurer, Jr. ${ }^{1}$, Derek L. G. Hill ${ }^{1}$, Robert J. Maciunas ${ }^{2}$, John A. Barwise ${ }^{2}$, \\ J. Michael Fitzpatrick ${ }^{2}$, and Matthew Y. Wang ${ }^{2}$ \\ 1 Division of Radiological Sciences and Medical Engineering, King's College London, UK \\ \{c.maurer, d.hill\}oumds.ac.uk \\ 2 Departments of Neurological Surgery (rjm), Anesthesiology (jab), and Computer \\ Science (jmf, myw), Vanderbilt University, Nashville, Tennessee
}

\begin{abstract}
We measured the deformation of the dura and brain surfaces between the time of imaging and the start of surgical resection for 21 patients. All patients underwent intraoperative functional mapping, allowing us to measure brain surface motion at two times that were separated by nearly an hour after opening the dura but before resection. The positions of the dura and brain surfaces were recorded and transformed to the coordinate space of a preoperative MR image using the Acustar neurosurgical navigation system. The mean displacements of the dura and the first and second brain surfaces were $1.2,4.4$, and $5.6 \mathrm{~mm}$, respectively, with corresponding mean volume reductions under the craniotomy of 6,22 , and $29 \mathrm{ml}$. The maximum displacement was greater than $10 \mathrm{~mm}$ in approximately one-third of the patients for the first brain surface measurement and one-half of the patients for the second. In all cases the direction of brain shift corresponds to a "sinking" of the brain intraoperatively, compared with its preoperative position. We observed two patterns of the brain surface deformation field depending on the inclination of the craniotomy with respect to gravity. Separate measurements of brain deformation within the closed cranium caused by changes in patient head orientation with respect to gravity suggested that less than $1 \mathrm{~mm}$ of the brain shift recorded intraoperatively could have resulted from the change in patient orientation between the time of imaging and the time of surgery. These results suggest that intraoperative brain deformation is an important source of error that needs to be considered when using neurosurgical navigation systems.
\end{abstract}

\section{Introduction}

It is becoming increasingly common for neurosurgical procedures to be performed with the assistance of a localizer and computer system that enables the surgeon to relate the position of a surgical instrument to structures of interest visible in preoperative images. Procedures performed using such systems are often described as image-guided surgery. The most established image-guided surgery systems use a stereotactic frame. More recently, a variety of frameless systems have been developed. Methods of physical space localization include articulated mechanical arms, ultrasonic range-finding systems, electromagnetic systems, and active and passive optical techniques. All current neurosurgical navigation systems assume that the head and its contents behave as a rigid body and use extrinsic features (skin-affixed or bone-implanted markers) or 
anatomical structures (point landmarks or surfaces) to determine the rigid-body registration transformation.

Errors in image-guided surgery caused by errors in identifying the external features used for registration, geometrical distortion in the preoperative images, and errors in the tracking of surgical instruments have been well documented for several systems $[3,7]$. Another potentially important source of errors is brain deformation between the time of imaging and the time of surgery or during surgery. Such deformations will invalidate the rigid-body assumption and consequently introduce inaccuracies into the system that will not be detected by the standard measures of registration or tracking error.

Surprisingly, little quantitative measurement of brain deformation has been published. A number of investigators have reported motion of brain structures while resection is underway. As far as we are aware, preliminary quantitative measurements were first reported by a number of groups, including us, at several recent conferences [1,2, $5,9]$.

In this study, which follows a pilot study of five patients [5], we measured the deformation of the dura and brain surfaces between the time of imaging and the start of surgical resection for 21 patients. All patients underwent intraoperative functional mapping prior to resection, providing us with the opportunity to measure brain surface shift at two times that were separated by nearly an hour after opening the dura but before performing surgical resection of the lesion. The positions of the dura and brain surfaces were recorded and transformed to the coordinate space of a preoperative MR image using the Acustar neurosurgical navigation system [7].

\section{Methods}

\subsection{Clinical Material}

We evaluated intraoperative brain surface movement by analyzing data obtained from 21 patients that underwent craniotomies for the resection of cerebral lesions at Vanderbilt University Medical Center between September 1996 and May 1997. Patients undergoing a second operation were excluded from consideration in this study. The cases included tumors that were located primarily in the left hemisphere (18 left, 3 right). The types of tumors were as follows: 13 low-grade gliomas, 5 anaplastic astrocytomas, 2 glioblastomas multiforme, and 1 teratoma. The tumor locations were as follows: 2 frontal, 5 temporal, 1 parietal, 6 fronto-temporal, 1 fronto-parietal, 4 temporo-parietal, 1 temporo-occipital, and 1 parieto-occipital. The patients ranged in age from 19 to 68 years (mean $\pm \mathrm{SD}=39 \pm 15 \mathrm{yr}$ ). The patients were operated on in the supine position with the head oriented $90^{\circ}$ right or left (for the left and right tumors, respectively) Functional mapping of sensory, motor, and/or language areas was performed using intraoperative cortical stimulation for all patients in this study. This provided an opportunity to measure brain surface movement at two times that were separated by nearly an hour after opening the dura but before performing surgical resection of the lesion.

The anesthesia was as standardized as possible. All patients received $20 \mathrm{mg}$ of dexamethasone every 3 hours beginning just before surgery. Mannitol ( $1 \mathrm{~g} / \mathrm{kg})$ was administered at the start of the creation of the craniotomy burr holes. Serum osmolality was 
generally in the 280-290 osmol range before mannitol administration and increased to 300-310 osmol after 30 minutes, at which point it remained fairly constant during the surface measurements. No patient was given narcotics or benzodiazepines. All patients received $60 \mathrm{ml}$ of $0.5 \%$ lidocaine with $0.125 \%$ bupivacaine applied to their scalp incisions and Mayfield clamp sites for local analgesia. Patients initially received propofol $(50-200 \mu \mathrm{g} / \mathrm{kg} / \mathrm{min}$ total anesthesia administered intravenously). The infusion was reduced to $25-75 \mu \mathrm{g} / \mathrm{kg} / \mathrm{min}$ at the start of the creation of the burr holes and was stopped when the section of cranium was elevated. Breathing resumed spontaneously after this through a laryngeal mask airway, which was removed when the patient responded to commands. The patients were aroused and awake by the time the section of cranium was elevated and for all surface measurements. Pulse and blood pressure were maintained within $20 \%$ of their preoperative values.

\subsection{Preoperative Image Acquisition}

The surgeries were performed using the Acustar neurosurgical navigation system (manufactured by Johnson \& Johnson Professional, Inc., Randolph, MA; the Acustar trademark is now owned by Picker International, Highland Heights, $\mathrm{OH}$ ) for intraoperative guidance [7]. Before the patient was imaged, the surgeon implanted four plastic posts into the outer table of the cranium of the patient, with one end remaining outside the skin. The specific locations of the posts were determined by individual clinical circumstances, but generally the posts were widely separated and placed on both sides of the head, with two of the markers inferior and two superior to the region of surgical interest. Image markers that contain contrast fluid and generate high intensity in both CT and MR images were attached to the posts just before image acquisition.

Both CT and MR images were acquired preoperatively for all patients (except that no CT image was acquired for one patient). Imaging studies were performed the day before or the morning of the surgical procedure. The CT images were acquired using a Siemens Somatom Plus scanner. Each image volume contained between 39 and 47 transverse slices with $512 \times 512$ pixels. The voxel dimensions were $0.4 \times 0.4 \times$ $3.0 \mathrm{~mm}$. All CT image volumes in this study were stacks of image slices with no interslice gap or slice overlap. The gantry tilt angle was zero. Three-dimensional (3-D) MP-RAGE MR image volumes were acquired using the head coil in a Siemens Magnetom SP4000 $1.5 \mathrm{~T}$ scanner. Each image volume contained 128 coronal slices with 256 $\times 256$ pixels. The voxel dimensions were typically $1.0 \times 1.0 \times 1.6 \mathrm{~mm}$. The readout gradient was oriented in the cranio-caudal direction with a magnitude of $4.7 \mathrm{mT} / \mathrm{m}$.

The centroid of each image marker was determined using the marker localization algorithm described in [11].

\subsection{Intraoperative Measurements}

Intraoperatively, the head was fixed in a Mayfield clamp, physical-space markers were attached to the marker posts, a localization probe was calibrated, and the markers were localized. The localization probe is equipped with an array of infrared emitting diodes (IREDs). Physical space tracking of the probe was accomplished with optical triangulation using an Optotrak 3020 (Northern Digital, Ontario, Canada). The physical-space 
markers are manufactured with a hemispherical divot, the center of which corresponds to the centroid of the image markers. The tip of the probe is a spherical ball. Intraoperative localization of each marker was performed by placing the ball-point tip into the divot and pressing a button on the probe handle.

The standard Acustar system registers images to each other and to the intraoperative physical coordinate system. When the localizer tip is placed near or within the patient, triplanar reformatted images intersecting at the corresponding position in image coordinates are generated. The Acustar system used for the work presented here was enhanced so that it could also record probe tip positions after registration was performed. To collect a dura or brain surface point, the surgeon placed the probe tip on the surface and recorded its 3-D coordinates by pressing a button. It took two or three seconds to acquire each point. The number of points collected in each acquisition ranged from 39 to 69 (mean $\pm \mathrm{SD}=56 \pm 7$ ). We attempted to collect surface points as uniformly distributed over the craniotomy as possible. We attempted to collect brain surface points on the gyral "envelope." Specifically, we avoided recording tip positions inside the sulci.

Dura surface points were collected soon after elevating the craniotomy. Points on the brain surface were collected twice, once after opening the dura but before functional mapping, and once after mapping but before performing surgical resection of the tumor. Immediately before every surface data acquisition, we recorded the position of a marker visible in the surgical field and collected approximately eight skull surface points around the edge of the craniotomy. To test the reproducibility of the surface data points, for four patients, we had two surgeons each collect a set of brain surface points, one immediately after the other.

\subsection{Data Analysis}

The distance between a dura, brain, or cranial surface point collected intraoperatively and the corresponding surface in the preoperative images was found by first transforming the physical space point to the image coordinate system using the rigid-body transformation provided by the Acustar system and then calculating the closest point on a triangle set representation of the image surface [6]. The image-to-physical rigid-body transformation was determined by fitting the image and physical space marker positions in a least-squares sense [7]. The dura and brain surfaces were manually delineated in the preoperative MR images using an interactive segmentation tool. The brain contours were drawn around the gyral envelope and did not invaginate into the sulci. Each resulting stack of polygonal surface contours was converted into a triangle set as described in [6]. A triangle set representation of the cranial surface was automatically extracted from each CT image using the tetrahedral decomposition method of Gueziec \& Hummel [4]. Because the localization probe tip is a spherical ball, the recorded position was systematically displaced from the true surface position by a distance equal to the radius of the spherical tip, which was $1.5 \mathrm{~mm}$ for all of the measurements made in this study. The calculated point-to-surface distances were positive if the intraoperatively recorded points were outside the brain image surface and negative if the points were inside the surface. The systematic displacement introduced by the finite diameter of the probe tip was corrected for by subtracting the tip's radius from these point-to-surface distances 
before further analysis. To test the reproducibility of dura and brain surface segmentation, we had two people each draw contours for four of the patients.

The craniotomy area was estimated from the skull surface points collected around the edge of the craniotomy as follows. The plane that best fit the skull points in a leastsquares sense was determined, the points were projected onto this plane, and the area of the polygon formed by these projected points was computed. For three patients, the craniotomy inclination was estimated by computing the angle between the normal to the plane fit through the skull points and the gravity field direction. The direction of gravity was estimated as the normal to a plane fit through approximately eight points collected on the surface of a pan of water placed within the optical field of view.

With the Acustar system, a "reference emitter" containing an array of IREDs is rigidly attached to the Mayfield head clamp via a multijointed arm. The reference emitter defines the intraoperative coordinate system and thus allows movement of the operating table and repositioning of the Optotrak 3020 when necessary, e.g., to maintain an optical line of sight. This technique does not compensate for patient movement relative to the Mayfield clamp. We estimated the magnitude of gross head motion relative to the Mayfield clamp between registration and the collection of a set of surface points in two ways: 1) by computing the distance between the position of a marker visible in the surgical field at the time of surface point collection and its position at the time of registration, and 2) by computing the distance between skull points collected around the edge of the craniotomy immediately before the dura or brain surface points were collected and a triangle set representation of the skull surface derived from the CT scans.

\subsection{Effect of Orientation}

Patients are normally operated on in a different position from that in which they are imaged. For example, all the patients studied here were imaged supine but operated on with their heads in a lateral position. To assess what proportion of the intraoperative brain shift we measured might be due to the change in brain orientation relative to gravity, we acquired MR images for two patients in both a conventional supine position and a prone position. We chose these two patient orientations because we were unable to get the patients positioned comfortably in the MR scanner in the operative position (head orientation $90^{\circ}$ left or right), and also because we believe that imaging a patient both prone and supine is likely to give an upper bound on the motion of the brain with respect to the cranium resulting from change in patient orientation.

We used three techniques to estimate the brain shift resulting from the change in patient orientation. The first technique is analogous to the method used to assess intraoperative shift. We manually delineated the brain surface from both scans using the Analyze software package (Biomedical Imaging Resource, Mayo Foundation, Rochester, $\mathrm{MN}$ ), and transformed the prone brain surface contours to the supine image coordinate space using the registration transformation calculated from the bone-implanted markers. Triangle set representations of each surface were created as described in Section 2.4 , and the difference in brain surface position was calculated for the portion of the cerebrum superior to the orbital-meatal line. The second technique is a comparison of the bone-implanted marker registration transformation ("cranial transformation") and a voxel-similarity registration transformation computed using the segmented 
brains ("brain transformation"). The voxel-similarity registration algorithm automatically finds the rigid-body transformation between the two segmented brains that maximizes the normalized mutual information of the joint probability distribution of the two images [10]. The discrepancy between these two transformations provides an estimate of the motion of the brain with respect to the skull caused by the change in patient orientation. We calculated the mean and maximum discrepancy in position of the brain voxels resulting from the difference in these transformations. Finally, we visually assessed brain deformation by computing thresholded boundaries in the prone images and overlaying them on the supine images, using both the cranial and brain transformations.

\section{Results}

\subsection{Intraoperative Brain Deformation}

The distance between the position of each dura and brain surface point collected intraoperatively with a localization probe and the nearest point on the same surface manually segmented from the preoperative MR images was calculated. Positive distances represented physical points outside the image surface and negative distances points inside the surface. Thus, negative distance values represented a "sinking" of surface points relative to the cranium, compared with their preoperative position. Similarly, positive values represented "bulging" or "protruding" points.

Because of the possibility that the patient may move with respect to the coordinate system defined by the reference emitter, e.g., within the Mayfield clamp, we verified that the registration was still accurate at the time of surface point collection. The two measures we used to assess the validity of the registration at the time of surface point collection were the distance between a marker visible in the surgical field and its position at the time of registration, and the distance of skull surface points from the skull surface segmented from a CT image. For two patients, both the marker displacement and mean skull point distance were greater than $1.5 \mathrm{~mm}$. For the remaining 19 patients, the mean marker displacement was $0.3-0.4 \mathrm{~mm}$, and the maximum displacement for all measurements was $0.8 \mathrm{~mm}$. The mean cranial point distance was $0.7-0.8 \mathrm{~mm}$, and the maximum distance was $1.2 \mathrm{~mm}$. These small numbers suggested that for these 19 patients, the image-to-physical transformation determined at the beginning of surgery was still accurate at the time of surface point collection.

Table 1 lists summary statistics of dura and brain surface displacement for these 19 patients. Dura surface points were collected soon after elevating the craniotomy. Brain surface points were collected after opening the dura but before performing cortical stimulation (Brain 1) and after stimulation but before performing surgical resection of the

tumor (Brain 2). The first and second sets of brain points were collected $53 \pm 27$ (mean $\pm \mathrm{SD}$ ) and $98 \pm 31$ minutes, respectively, after the dura points were collected. The 10 and $90 \%$ distance values were determined by sorting the distances in descending order and taking the $(0.10 n)$ th and $(0.90 n)$ th elements in the sorted list, where $n$ is the number of values (one for each point). The 10 and $90 \%$ distance values approximated the range of brain movement. We used them rather than the minimum and maximum values to eliminate the possibility that the extreme values represented erroneous outliers. The three displacements (dura and two brain surfaces) were significantly different from 
Table 1. Intraoperative Dura and Brain Surface Displacement (mm)

\begin{tabular}{|c|l||c|c|c|}
\hline & Surface & Mean & $10 \%$ & $90 \%$ \\
\hline \hline \multirow{3}{*}{ Mean \pm SD } & Dura & $-1.2 \pm 2.0$ & $0.5 \pm 1.6$ & $-3.0 \pm 2.5$ \\
\cline { 2 - 5 } & Brain 1 & $-4.4 \pm 1.9$ & $-1.0 \pm 1.1$ & $-7.9 \pm 3.2$ \\
\cline { 2 - 5 } & Brain 2 & $-5.6 \pm 1.9$ & $-1.2 \pm 1.1$ & $-10.3 \pm 3.2$ \\
\hline \hline \multirow{3}{*}{ Min to Max } & Dura & 2.0 to -4.8 & 3.4 to -1.9 & 0.9 to -8.1 \\
\cline { 2 - 5 } & Brain 1 & -1.3 to -7.1 & 2.8 to -2.5 & -2.3 to -13.2 \\
\cline { 2 - 5 } & Brain 2 & -3.1 to -8.5 & 2.4 to -3.0 & -6.2 to -15.0 \\
\hline
\end{tabular}
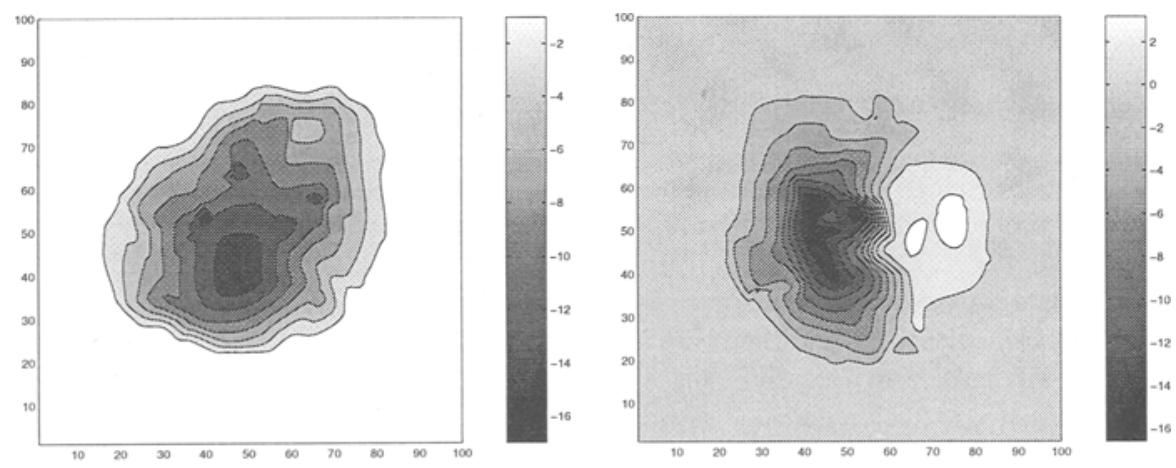

Fig. 1. Plots showing the spatial distribution of brain surface movement.

each other (two-tailed paired $t$ test, $P<0.01$ ). This is true for both the mean and $90 \%$ displacement values. The $90 \%$ displacement was greater than $10 \mathrm{~mm}$ in approximately one-third of the patients ( 7 of 19 patients) for the first brain surface and approximately one-half of the patients ( 10 of 19 patients) for the second.

Figure 1 shows contour plots of brain surface displacement. The left plot was created from data regarding a patient whose craniotomy inclination was 6 degrees, and is typical of most of the patients in this study. The brain was sinking under the craniotomy relative to its preoperative position. The displacement field was shaped somewhat like a bowl, with the largest displacement near the center of the craniotomy. The right plot was created from data regarding a patient whose craniotomy inclination was 34 degrees. Again the brain was sinking over much of the area of the craniotomy, but there was a region that was bulging or protruding (positive displacement values). This protruding region was at the lowest edge of the craniotomy. A similar pattern of bulging at the gravitationally dependent edge was observed in two other patients. Figure 2 shows the intraoperatively recorded brain surface overlayed as a white line on coronal slices from the preoperative MR image volumes for the same two patients shown in Fig. 1.

To test the reproducibility of dura and brain surface segmentation, for four patients, we had two people each draw contours. The mean distance between the surfaces obtained by each person ranged from -0.6 to $0.5 \mathrm{~mm}$ for dura and -0.4 to $0.5 \mathrm{~mm}$ for brain. The overall means were 0.1 and $0.2 \mathrm{~mm}$ for dura and brain, respectively. To test 

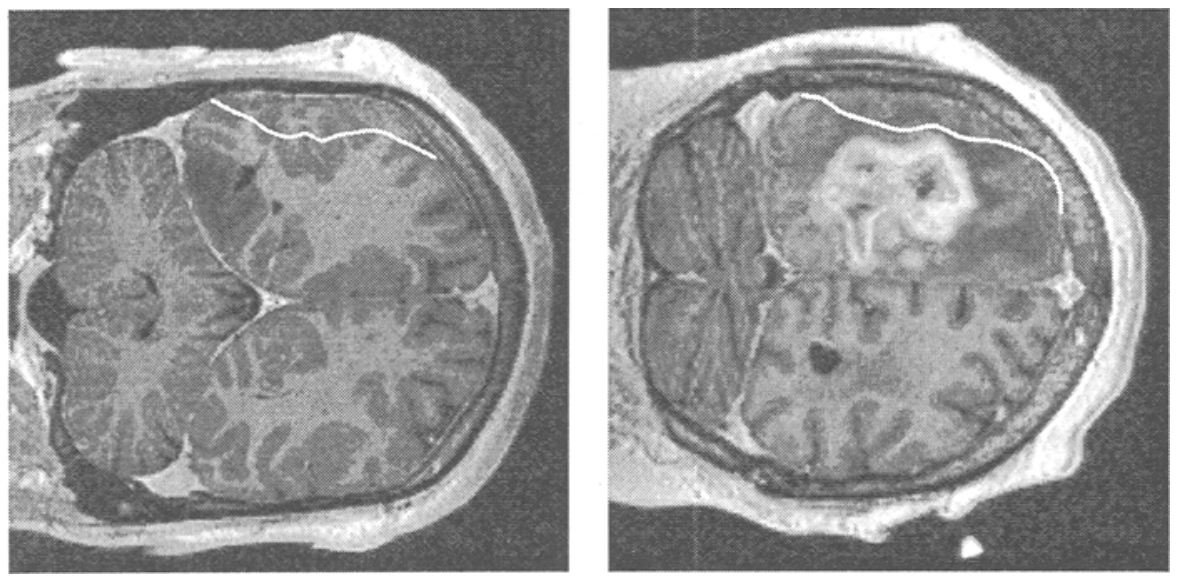

Fig. 2. Coronal image slices from the MR image volumes of two patients, showing the intraoperatively recorded brain surface overlayed as a white line. These images correspond to the data presented in Fig. 1. The image slices have been rotated to indicate the intraoperative orientation of the patient, with the direction of gravity vertical on the page. The patient's left side is at the top in each image. The ends of the white lines represent the edges of the craniotomy. In both patients, the brain was sinking under much of the craniotomy, but in the patient shown on the right, there was a slightly protruding region at the lowest edge of the craniotomy.

the reproducibility of the measurement of intraoperative brain surface movement, we had two surgeons each collect a set of brain surface points, one immediately after the other. Brain surface displacement was calculated for each set of measurements. The difference in mean brain surface displacement obtained by each surgeon ranged from -0.4 to $0.3 \mathrm{~mm}$. The difference in overall means was $0.1 \mathrm{~mm}$.

Patient age, craniotomy size, mannitol dose, time since mannitol infusion, net fluid volume change, and partial pressure of arterial carbon dioxide might all contribute to the amount of dura and brain surface shift. We examined plots of and calculated linear regressions of surface point displacement as compared with these variables. There were no visually obvious trends and no statistically significant correlations.

\subsection{Effect of Orientation}

The effect of orientation on the deformation of the brain within the closed cranium was assessed for two patients who were imaged prone and supine. Brain deformation between these imaging studies was quantified using the three techniques described in Section 2.5. The mean and SD of the brain surface displacement calculated using the first technique were less than $1 \mathrm{~mm}$ for both patients. Using the second technique, we determined that the mean discrepancy between the cranial and brain transformations for the two patients was less than $0.5 \mathrm{~mm}$ and that the maximum discrepancy was less than $1 \mathrm{~mm}$. Both sets of measurements suggested that motion of the brain relative to the cranium resulting from a change in patient orientation only (i.e., without a craniotomy) is less than $1 \mathrm{~mm}$. The mean discrepancies we obtained were only slightly larger than 

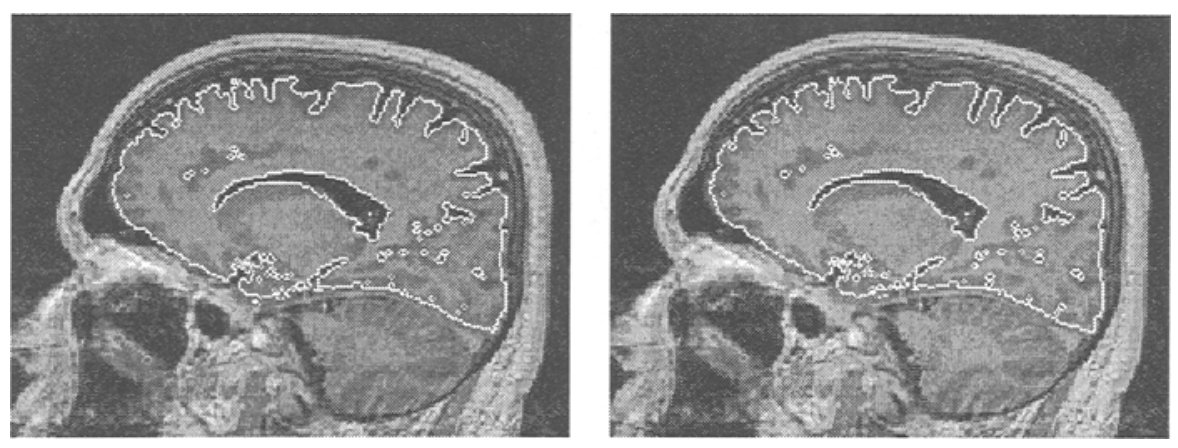

Fig. 3. Visual assessment of the effect of head orientation on brain shift. Threshold boundaries were computed in the prone images and overlayed on the supine images. In the left and right columns, boundaries were mapped using the cranial and brain transformations, respectively. There was no visually apparent brain shift using either transformation.

the expected error of the bone-implanted marker system, so the actual brain shift may have been smaller than these upper-bound figures suggest. Figure 3 shows the threshold boundary overlays for one of the two patients. There is no visually apparent brain shift using either the cranial or brain transformation.

\section{Discussion}

\subsection{Sources of Error}

There are several sources of error in a surgical navigation system: error inherent in the registration process, geometrical distortion in the images, movement of the patient with respect to the system during surgery, and movement of the brain relative to the cranium between scanning and the time of surgery. Because the purpose of this paper is to measure the latter, we need to examine the magnitude of the former.

The accuracy of the Acustar system was determined, in an earlier clinical trial, to be $1.0 \pm 0.5 \mathrm{~mm}$ (mean $\pm \mathrm{SD}$ ) for CT-physical registration and $1.3 \pm 0.6 \mathrm{~mm}$ for MRphysical registration [7]. These values include error in localizing the marker positions, error in tracking (i.e., finding the position of) the localization probe, and geometrical distortion in the image. The image-to-physical registrations using MP-RAGE MR images in the current study are likely to have been more accurate than the registrations obtained using the spin-echo MR images that were used in our previous studies [7].

The mean degradation in accuracy of the Acustar system during surgery was determined, in an earlier study, to be approximately $0.5 \mathrm{~mm}$, with a change greater than $1.5 \mathrm{~mm}$ observed in 3 of 24 patients [7]. We believe that such head movement, some of which may occur during elevation of the craniotomy, is the principal cause of the degradation in accuracy. If the measured displacement of the brain were caused by head motion relative to the Mayfield clamp, then the bone surface and brain surface would be displaced in the same way. Thus, in this study, at the time of surface point 
collection, we measured the displacement of a marker visible in the surgical field relative to its position at the time of registration and calculated the distance of skull surface points from the skull surface segmented from a CT image. In 2 of 21 patients, both marker displacement and mean skull point distance were greater than $1.5 \mathrm{~mm}$. In the remaining patients, the mean (maximum) marker displacement was $0.3-0.4(0.8) \mathrm{mm}$ and skull point distance was $0.7-0.8$ (1.2) mm. For these 19 patients, the image-tophysical transformation determined at the beginning of surgery was still accurate at the time of surface point collection.

Surface segmentation error and intraoperative surface point localization error also contribute to brain shift measurement error. We assessed the reproducibility of both manual delineation of the brain surface from the MR images and intraoperative surface point collection and determined that different observers produced results that differed by less than $0.5 \mathrm{~mm}$ for both measurements. There is also the possibility of bias in our surface measurements resulting from the finite size of the spherical probe tip and the depression of the brain surface when recording measurements with the probe. The small values of the skull surface point distances after correcting for the size of the probe tip and the very small brain shifts measured at the edge of the craniotomies (see Figs. 1 and 2) suggested that our surface measurements are substantially free of bias.

It is important to note that we calculated the distance between a surface point collected intraoperatively and the nearest point on the same surface manually segmented from the preoperative MR image. The nearest point on the segmented surface was not necessarily the corresponding point, and thus, our displacement values are clearly lower bound estimates. In summary, the cortical surface shifts we calculated were substantially higher than our measurement error. The actual shifts are probably higher than our calculated shifts because we were using nearest image surface points rather than corresponding points.

\subsection{Possible Causes of Brain Shift}

We were surprised by the magnitude of the brain surface shift that we recorded. Exploring the reasons for the shift might make it possible to develop enhancements to surgical navigation systems to enable them to compensate for it.

High speed MR imaging techniques have previously been used to measure brain motion during imaging [8]. Pulsatile motion with the same period as the cardiac cycle and an amplitude of up to $0.5 \mathrm{~mm}$ was reported. The intraoperative brain shift we measured in our study was an order of magnitude larger than the reported pulsatile motion. The intraoperative brain surface deformation was also substantially larger than shift caused by a change in the orientation of the patient's head between the time of imaging and the time of surgery.

It is standard surgical practice to reduce intracranial pressure (ICP) before performing neurosurgical procedures. Steroids are often administered preoperatively to reduce inflammation. Intraoperatively, cerebral blood volume can be controlled by manipulating ventilation to alter carbon dioxide concentration in the blood and by tilting the bed to increase or reduce venous drainage. Cerebrospinal fluid (CSF) volume can be altered by reducing CSF production or by draining CSF. The water content of the brain can be reduced by administering an osmotically active drug, e.g., the sugar alcohol mannitol. 
The effect these parameters have on ICP is well documented, but little is known about the resulting volume changes and brain deformation they cause in humans.

We approximated the volume between the brain surface in the image and the brain surface recorded intraoperatively by integrating the volume under surface displacement plots such as those shown in Figs. 1. This provides a reasonable approximation to the volume change under the craniotomy because the craniotomies are relatively flat and because the displacements are approximately normal to the plane of the craniotomy. This estimate does not include any volume change associated with brain shift outside the craniotomy. The mean \pm SD volume changes under the craniotomy were $-6 \pm 11$, $-22 \pm 10$, and $-29 \pm 11 \mathrm{ml}$ for the dura, first, and second brain surfaces, respectively. The mean change for the dura surface was less than $1 \%$ of typical brain volume. The mean changes for the brain surfaces were approximately $1-2 \%$.

\subsection{Consequences for Image-Guided Surgery}

Motion of the brain surface relative to the cranium may not be an important factor in some types of neurosurgery. For example, if a lesion of interest is at the base of the cranium, surrounded by cranial nerves and blood vessels, it may move much less relative to the skull than the brain surface immediately underneath a craniotomy. Our measurements are unlikely to have much applicability for assessing errors at such deep structures. Furthermore, we measured brain motion only in the direction perpendicular to the brain surface. This type of motion has only a minor influence on the accuracy of image-guided surgery for planning the position of a craniotomy.

The motion that we measured in this study will directly affect the accuracy with which a surgeon can judge his or her depth into the brain in the vicinity of the surface. For example, if the surgical navigation system is being used to assist the surgeon in identifying the distal edge of a superficial lesion, then brain motion in the direction perpendicular to the brain surface directly degrades the accuracy of the system.

The goal of this study was to accurately quantify intraoperative deformation of the brain cortical surface. Clearly the ultimate goal of this field of research is to quantify 3-D brain deformation. Bucholz [1] recently reported some preliminary studies of subsurface structure displacement using intraoperative ultrasonography. The brain cortical surface is a good starting point for measuring subsurface deformation because the surface is visible and it is possible to obtain very accurate measurements of the surface. Intraoperative ultrasound images typically have a low signal-to-noise ratio, and segmentation of subsurface structures is often difficult. It might be possible to quantify 3-D brain motion using elastic deformation models. Measurements of surface displacement similar to those we have reported in this study can potentially be used as boundary conditions for such models. Further work is necessary to quantify the deformation of subsurface structures and to determine how deformation is influenced by the resection process. It is possible that further studies will find that for some types of neurosurgical procedures requiring high accuracy, brain deformation is substantial and too variable to be corrected using computational algorithms. If this turns out to be the case, then accurate imaging guidance for these procedures could be provided only by high-quality intraoperative imaging, such as interventional MR imaging. 
Any evidence of brain motion derived from this study, especially in the context of otherwise satisfactory deep brain target localization, must not be used to justify inaccurate systems or pessimism about system accuracy in general. Instead, it should prompt renewed efforts to develop techniques to minimize this motion or correct for it dynamically in order to achieve better registration.

\section{References}

1. Bucholz RD, Yeh DD, Trobaugh J, et al: The correction of stereotactic inaccuracy caused by brain shift using an intraoperative ultrasound device. In: Troccaz J, Grimson E, Mösges R (eds): CVRMed-MRCAS '97, Berlin, Springer-Verlag, 1997, pp 459-466.

2. Dorward NL, Alberti O, Velani B, et al: Early clinical experience with the EasyGuide neuronavigation system and measurement of intraoperative brain distortion. In: Hellwig D, Bauer BL (eds): Minimally Invasive Techniques for Neurosurgery, Heidelberg, Springer-Verlag, 1997, pp 193-196.

3. Golfinos JG, Fitzpatrick BC, Smith LR, Spetzler RF: Clinical use of a frameless stereotactic arm: Results of 325 cases. J Neurosurg 83:197-205, 1995.

4. Gueziec A, Hummel R: Exploiting triangulated surface extraction using tetrahedral decomposition. IEEE Trans Visualization Comput Graph 1:328-342, 1995.

5. Hill DLG, Maurer CR Jr, Wang MY, et al: Estimation of intraoperative brain surface movement, In: Troccaz J, Grimson E, Mösges R (eds): CVRMed-MRCAS '97, Berlin, SpringerVerlag, 1997, pp 449-458.

6. Maurer CR Jr, Aboutanos GB, Dawant BM, et al: Registration of 3-D images using weighted geometrical features. IEEE Trans Med Imaging 15:836-849, 1996.

7. Maurer CR Jr, Fitzpatrick JM, Wang MY, et al: Registration of head volume images using implantable fiducial markers. IEEE Trans Med Imaging 16:447-462, 1997.

8. Poncelet BP, Wedeen VJ, Weisskoff RM, Cohen MS: Brain parenchyma motion: Measurement with cine echoplanar MR imaging. Radiology 185:645-651, 1992.

9. Reinges MHT, Krombach G, Nguyen H, et al: Assessment of intraoperative brain tissue movements by frameless neuronavigation. Comput Aided Surg 2:218, 1997 (abstract).

10. Studholme C, Hill DLG, Hawkes DJ: Automated 3D registration of MR and PET brain images by multi-resolution optimisation of voxel similarity measures. Med Phys 24:25-35, 1997.

11. Wang MY, Maurer CR Jr, Fitzpatrick JM, Maciunas RJ: An automatic technique for finding and localizing externally attached markers in CT and MR volume images of the head. IEEE Trans Biomed Eng 43:627-637, 1996. 